\title{
Meta
}

Journal des traducteurs

Translators' Journal

\section{ZARANDONA, Juan Miguel (ed.) (2014) : De Britania a Britonia. La leyenda artúrica en tierras de Iberia : cultura, literatura y traducción. Berna: Peter Lang, 458 p.}

\section{Cristina Adrada Rafael et Marie-Hélène García}

Volume 62, numéro 1, avril 2017

URI : https://id.erudit.org/iderudit/1040474ar

DOI : https://doi.org/10.7202/1040474ar

Aller au sommaire du numéro

Éditeur(s)

Les Presses de l’Université de Montréal

ISSN

0026-0452 (imprimé)

1492-1421 (numérique)

Découvrir la revue

Citer ce compte rendu

Adrada Rafael, C. \& García, M.-H. (2017). Compte rendu de [ZARANDONA, Juan Miguel (ed.) (2014) : De Britania a Britonia. La leyenda artúrica en tierras de Iberia : cultura, literatura y traducción. Berna : Peter Lang, 458 p.] Meta, 62(1), 223-224. https://doi.org/10.7202/1040474ar d'utilisation que vous pouvez consulter en ligne. 


\section{DOCUMENTATION}

Zarandona, Juan Miguel (ed.) (2014): De Britania a Britonia. La leyenda artúrica en tierras de Iberia: cultura, literatura y traducción. Berna: Peter Lang ${ }^{1}, 458$ p.

La figure du roi Arthur, infatigable, nous parvient de sa Britannia natale à la péninsule ibérique grâce à Juan Miguel Zarandona, enseignant-chercheur à l'Université de Valladolid, qui, dans le cadre de son projet de recherche Clytiar (Culture, littérature et traduction ibéro-arthurienne), a coordonné et dirigé cet ouvrage, publié aux éditions Peter Lang, dans lequel lui et ses collaborateurs nous font revivre le développement de la légende arthurienne dans la péninsule ibérique à travers des créations artistiques ou littéraires.

Comme le coordinateur nous l'annonce dans l'introduction, ce livre nous invite à embarquer pour un voyage unique à travers les terres ibériques, nous transportant jusqu'à des veillées autour du feu pour écouter les légendes de la Table Ronde, du Roi Arthur ou de Merlin l'Enchanteur, à la cour de Camelot. Ce voyage se fait dans l'ordre chronologique, de la légende la plus ancienne, dans le premier chapitre, à la plus contemporaine, dans le dernier. Une réussite qui contribue sans aucun doute à insuffler de la réalité à la fiction.

En effet, les aventures et mésaventures de ce chevalier - ni cervantin ni castillan - certes, mais très populaire en Espagne, ont suscité un grand intérêt chez de nombreux auteurs. Et pour le prouver, comme il ne pouvait en être autrement, ce volume traite de la littérature arthurienne en adoptant une perspective multiséculaire et interlinguistique, tant par les langues concernées dans chaque étude que par la langue de rédaction des chapitres (espagnol, galicien, catalan et portugais).

C'est ainsi que le lecteur le perçoit dès le début, grâce à un prologue soigneusement rédigé en anglais par Kevin J. Harty, immédiatement suivi d'une traduction en espagnol réalisée par Juan Manuel García González.

Dans le premier chapitre, écrit en portugais par Ana Margarida Chora, de l'Université de Lisbonne, "Lais da Bretanha de Lançarot e Marot e os episódios correspondentes da Vulgata e Post-Vulgata (XIV)», l'auteure, dans une étude comparée de textes du XIII ${ }^{\mathrm{e}}$ siècle, cerne le rôle des «lais» dans la traduction arthurienne portugaise et ainsi l'importance de la production arthurienne sur le territoire portugais.

La seconde contribution nous vient de Carlos A. Sanz Mingo, de l'Université de Cardiff, «Un texto galés en España: la recepción y traducción de 'Culhwuch ac Olwen' de los Mabinogion (13501410)». Il nous propose ici une analyse des problèmes que présente la traduction de ce conte gallois en espagnol et il cherche à élucider les raisons de son faible succès en Espagne, car il ne compte que quatre traductions en castillan, la première ne datant que de la fin du $\mathrm{xx}^{\mathrm{e}}$ siècle.

José Ramón Trujillo, de l'Université autonome de Madrid, est l'auteur de l'étude, «Traducciones y refundiciones de la prosa artúrica en la península ibérica (XIII-XVI)», dans laquelle il nous présente une vision de cette littérature dans la péninsule, mettant en relief, comme facteur fondamental de pénétration dans les royaumes ibériques, le rôle important de la traduction des grands cycles en prose rédigés en France à partir du XIII ${ }^{\mathrm{e}}$ siècle.

Des royaumes ibériques nous nous déplaçons jusqu'à la Seigneurie de Biscaye avec l'étude de Juan Luis Ramos Merino, de l'Université d'Alcalá de Henares, "Merlín, Arturo y Jaun Zuria en la Vizcaya medieval: la reelaboración de un mito cultural europeo (XV)». Il reprend la figure du premier seigneur de Biscaye pour illustrer comment la chronique locale, historique et politique peut être enrichie - et conditionnée - par le mythe littéraire, comme cela advint au chroniqueur Lope García de Salazar au $\mathrm{Xv}^{\mathrm{e}}$ siècle, en assimilant et en adaptant les rôles des principaux personnages de la célèbre «littérature de Bretagne» pour enrichir la figure de Jaun Zuria, devenu un héros revêtant les attributs d'un chevalier idéal.

L'importance de la littérature arthurienne au-delà de l'Europe est attestée dans le chapitre suivant, «El personaje de Galván en el Baladro del sabio Merlín (1498) y la Demanda del Sancto Grial (1535)», écrit par Rosalba Lendo, de l'Université nationale autonome du Mexique. Elle nous y propose une analyse approfondie du personnage de Galván, neveu préféré du Roi Arthur et vaillant chevalier, et de la dégradation dont il souffrira à travers les différentes narrations des cycles en prose du XIII ${ }^{\mathrm{e}}$ siècle.

Notre itinéraire nous retient sur les terres américaines avec la contribution de Mario Botero García, de l'Université d'Antioquia (Colombie) qui, dans le texte "A propósito de la muerte de los amantes: Tristan en prose (ca. 1230-1235) et Tristán de Leonís (1501)», analyse le binôme amour et mort dans les figures de Tristan et Yseult grâce à ces deux œuvres, représentatives respectivement des traditions française et castillane. 
Après cette brève incursion sur les territoires ibéroaméricains, nous revenons en Europe avec Sebastián García Barrera, de l’Université de Rouen. Dans «Amadís en Francia, o el destino de una materia medieval en rumbo hacia el Renacimiento (1508) », il nous offre un approfondissement de la figure de l'Amadís de Gaula et de son importance en France, grâce à une analyse comparée de l'ouvrage espagnol de Rodríguez de Montalvo et de sa refonte française par Herberey des Essarts.

Le chapitre huit nous donne l'occasion de faire un nouveau saut temporel et d'emprunter des chemins plus modernes et contemporains. «Reliquias sagradas: viajeros y el Santo Grial (XIX)», rédigé par Roger Simpson, de l'Université d'East Anglia (Royaume-Uni), et traduit par Antonio Fernández Lucas, nous emmène dans l'univers du Saint Graal, toujours relié à la légende arthurienne, et réalise un parcours à la frontière entre imaginaire et réalité en analysant les reliques auxquelles fut attribué le pouvoir de cet objet magique.

Les deux contributions suivantes nous arrivent de nouveau des terres américaines, plus précisément des États-Unis, et reposent sur le même fil conducteur: le reflet de la légende de Tristan dans le roman des $\mathrm{XIX}^{\mathrm{e}}$ et $\mathrm{XX}^{\mathrm{e}}$ siècles. D'un côté, la contribution de Joan Grimbert, de l'Université catholique d'Amérique, intitulée «La Tristana de Galdós como subversión de la leyenda de Tristán (1892)», traduit par Sheila Daroca Narro, nous offre une vision intéressante et non moins originale de la protagoniste de Galdós comparée à la figure de Tristan; elle justifie comment ce dernier est présent dans l'œuvre du XIX ${ }^{\mathrm{e}}$ siècle. De son côté, Lisa Nalbone, de l'Université de Floride centrale, adopte la même approche mais avec un roman du $\mathrm{Xx}^{\mathrm{e}}$ siècle, traduit par José Gabriel Rodríguez Pazos, dans le chapitre "Conjuros, seducción y tribulaciones en La última fada de Emilia Pardo Bazán (1916)».

C'est la légende d'Excalibur qui suit celle de Tristan, dans une étude conjointe d'Isabel de Riquer Permanyer et de Daniel Navarro Torró, tous deux de l'Université de Barcelone. Écrite en catalan, "Art, tradició i llegenda en l'Escalibor riqueriana» (1910), elle permet aux auteurs de réaliser une étude de sa réception en Catalogne en prenant comme référence le poème original catalan d'Alexandre de Riquer.

De la littérature, nous passons à l'univers de la peinture, au chapitre douze, écrit par Alma Idamons (chercheure non rattachée à une institution). Dans «Salvador Dalí, pintor artúrico. La materia de Bretaña en su ballet Tristan Fou (1938-1944)», elle effectue une analyse minutieuse afin de relier et de justifier la présence arthurienne dans cette œuvre de l'excentrique et mondialement connu peintre espagnol.
Le chapitre suivant, écrit en galicien par Luis Martínez-Risco Daviña, de la Fundación Vicente Risco: «Vicente Risco e o mundo artúrico. Unha aproximación (1919-1990)», qui nous propose une sélection de textes inédits de l'écrivain et essayiste d'Ourense. Il est suivi de la contribution d'Almudena María Gómez Seoane, de l’Université de Saint-Jacques-de-Compostelle, qui nous fait rester dans la fiction littéraire de Galice, en prenant comme référence l'un des grands auteurs galiciens du $\mathrm{xx}^{\mathrm{e}}$ siècle, Álvaro Cunqueiro: «Importación y adaptación de la materia de Bretaña en la narrativa de ficción de Álvaro Cunqueiro: el ejemplo de la reina Ginebra en Merlín y Familia (1955)».

Enfin, la dernière touche apportée à ce voyage à travers les territoires ibériques et américains nous est fournie par le coordinateur de l'ouvrage, Juan Miguel Zarandona, dans le chapitre «Estudio comparado de los pseudogriales neomedievales contemporáneos de Paloma Díaz-Mas (1984) y Umberto Eco (2000): de la esperanza medieval a la parodia moderna». L'auteur se concentre ici sur Baudolino d'Eco et El rapto del Santo Grial o El caballero de la Verde Oliva de Díaz-Mas, dans lequel comme son titre l'indique, il compare la vision originelle, plus sérieuse, du Graal et celles proposées par ces deux écrivains, contemporaines et plus satiriques.

Il s'agit donc d'un volume méritant une lecture attentive, non seulement pour son intérêt scientifique mais aussi parce qu'il invite le curieux ou l'amateur de la légende arthurienne à la délectation. Il faut également féliciter le coordinateur, qui nous offre un livre dont le soin et la minutie, tant dans l'excellente qualité des contributions sélectionnées que dans l'organisation thématique et la présentation, nous rendent plus accessible le chemin initié vers les terres de la Britannia médiévale.

\section{Cristina Adrada Rafael Universidad de Valladolid, Valladolid, Espagne \\ MARIE-HÉLÈnE GARCÍA Université d'Artois, Arras, France}

\section{NOTE}

1. L'ouvrage est également disponible au format e-book (ISBN 978303510759 3).

Berry, Edward et Gémar, Jean-Claude (2010): La rédaction des motifs: manuel à l'usage des juges. Montréal: Éditions Thémis, 241 p.

Rendre la justice et laisser croire que justice a été faite (MacMillan 1948, cité dans Berry). Communiquer et persuader. Conscience et présence. Voilà tout l'art de la rédaction des motifs. Des principes 